\title{
ADECUACIÓN DEL LENGUAJE EN EL MATERIAL DIDÁCTICO ESCRITO: EL PROCESO DE PRODUCCIÓN COMO PROCESO PEDAGÓGICO
}

ADAPTATION OF LANGUAGE IN WRITTEN TEACHING MATERIALS: PRODUCTION PROCESS AS EDUCATIONAL PROCESS

Volumen 11, Número 2

pp. 1-14

Este número se publicó el 30 de agosto de 2011

Patricia Gómez Figueroa

María Benavides González

La revista está indexada en los directorios:

LATINDEX, REDALYC, IRESIE, CLASE, DIALNET, DOAJ, E-REVIST@S,

La revista está incluida en los sitios:

REDIE, RINACE, OEI, MAESTROTECA, PREAL, HUASCARAN, CLASCO 


\title{
ADECUACIÓN DEL LENGUAJE EN EL MATERIAL DIDÁCTICO ESCRITO: EL PROCESO DE PRODUCCIÓN COMO PROCESO PEDAGÓGICO
}

ADAPTATION OF LANGUAGE IN WRITTEN TEACHING MATERIALS: PRODUCTION PROCESS AS EDUCATIONAL PROCESS

\author{
Patricia Gómez Figueroa ${ }^{1}$ \\ María Benavides González²
}

\begin{abstract}
Resumen: El presente ensayo surge como respuesta a una serie de reflexiones sobre el quehacer de los editores académicos que laboran en el programa de Producción de Material Didáctico Escrito (Promade) de la Dirección de Producción de Materiales de Didácticos de la Universidad Estatal a Distancia (UNED). Se pretende un acercamiento al material escrito a través del lenguaje y los diferentes elementos que confluyen en su producción, como la situación comunicativa y la mediación pedagógica. Finalmente, se concluye con una serie de recomendaciones acerca de la relevancia de la adecuación del lenguaje como parte del proceso pedagógico en la producción del material didáctico.
\end{abstract}

Palabras clave: EDUCACIÓN A DISTANCIA, COMUNICACIÓN ESCRITA, ADECUACIÓN DEL LENGUAJE, PRODUCCIÓN DE MATERIAL DIDÁCTICO ESCRITO, COSTA RICA.

Abstract: This essay reflects on the work of the production editor of the program "Producción de Materiales Didácticos (Promade) de la Universidad Estatal a Distancia (UNED)". We present, therefore, some aspects related to the production area: written materials, mainly printed and main elements, like style, that come together in its development; and other like the communicative situation and pedagogical mediation. We conclude with a series of recommendations about the relevance of an adequate language an style as part of the educational process in the production of materials.

Key words: DISTANCE LEARNING, WRITTEN COMMUNICATION, LANGUAGE ADAPTATION, WRITTEN TEACHING MATERIALS, COSTA RICA

\footnotetext{
${ }^{1}$ Máster en Ecología y Fisiología Vegetal, Universidad de Costa Rica. Editora académica, Producción de Material Didáctico Escrito (Promade) de la Universidad Estatal a Distancia (UNED). Dirección electrónica:pgomez@uned.ac.cr

${ }^{2}$ Bachiller en Filología Española, Universidad de Costa Rica. Editora académica, Producción de Material Didáctico Escrito (Promade) de la Universidad Estatal a Distancia (UNED). Dirección electrónica: mbenavides@uned.ac.cr
}

Artículo recibido: 31 de mayo, 2011

Aprobado: 18 de agosto, 2011 


\section{Introducción ${ }^{3}$}

Al referirnos a la educación, un fenómeno que se establece como una disciplina en la medida en que se estudian sus componentes, es necesario considerarla como un proceso multidireccional mediante el cual se transmiten conocimientos, valores, costumbres y formas de actuar. Esto es educación: formar, desarrollar y capacitar los diferentes aspectos de la personalidad del individuo. El conjunto de leyes, normas o principios que se encargan de regular el proceso educativo, en un contexto histórico particular, se conoce como pedagogía (Castiñeiras, 2003).

Justamente, este proceso se experimenta no solo a través de palabras, sino de acciones, actitudes y sentimientos dando como resultado un proceso de vinculación y concienciación cultural, moral y conductual en el individuo (Thinès y Lempereur, 1978).

De esta manera, es por medio de la educación, y en nuestro caso particular a través de la educación a distancia, que las nuevas generaciones asimilan, aprenden los conocimientos, las normas de conducta, la manera de ser y las formas de ver el mundo, esta afirmación se señala, igualmente, en el Modelo Pedagógico de la Universidad Estatal a Distancia, a saber

En esta interiorización o asimilación tienen importancia central los procesos mediadores, ligados al contexto, el principal de los cuales lo constituyen los otros miembros del colectivo social y cultural. El mediador por excelencia es el medio simbólico, es decir, el lenguaje, que se utiliza en la comunicación e intercambio tanto de los conocimientos y conceptos, como de las estrategias y capacidades necesarias para su elaboración. (UNED, 2007, p. 34)

D'Alton (1994) afirma que el lenguaje humano, el cual no puede separarse de la inteligencia, se basa en la habilidad del individuo de comunicarse por medio de signos. Consiste en un conjunto de signos, orales y escritos, que por medio de su significado y su relación facilitan la expresión y la comunicación humana (Ruiz y Escandell, 2009).

3 Nota aclaratoria: Antes de iniciar propiamente con el desarrollo del tema, debemos aclarar que en el texto se utilizarán muchos conceptos tradicionales respecto a la educación, debido a que nuestras reflexiones parten de interrogantes surgidas del Modelo Pedagógico de la Universidad Estatal a Distancia, documento en el cual se mezclan diversos pensamientos, en muchos casos contradictorios, respecto a la educación, es decir, en la UNED no se da un modelo sino varios modelos pedagógicos o un collage de teorías de la educación. 
Así, precisamente, el alumno incorpora en su sistema conceptual propio nuevos significados, originados, también, por las palabras; como consecuencia de esta adquisición de nuevas nociones, el estudiantado debe asimilar tanto los conceptos como las prácticas discursivas de las disciplinas que aprende.

Aunado a lo anterior, la escritura, un signo escrito, es un poderoso instrumento que permite representar, analizar, revisar y transformar el conocimiento (Thurn, 1999). Por ello, es conveniente que los estudiantes saquen provecho de este potencial de conocimiento que condiciona las formas de comprender e interpretar el mundo y se ocupan de apoyar intensivamente la producción escrita que se les facilita por medio del texto y de diferentes apoyos multimediales.

El Modelo pedagógico de la Universidad Estatal a Distancia permite reflexionar acerca de factores clave, como el contexto y el lenguaje, este último como mediador por excelencia en el proceso educativo. Lo planteado se justifica al considerar cómo, aún en medio de los avances tecnológicos aplicados a la enseñanza, siguen vigentes los materiales didácticos impresos; sin embargo, advierte Fung

With developments in the use of information and communication technology in distance education, the recent literature in this area has been skewed towards studies focusing on online or web-based learning. However, research on print materials - particularly on ways of achieving goals in teacher education-should not be neglected at a time of increasing use of distance education in teacher education (...). (2005, p. 182) ${ }^{4}$

Por consiguiente, en respuesta al mencionado modelo pedagógico a distancia, el lenguaje y el discurso particular a los cuales se recurra deben ser los adecuados para el desenvolvimiento profesional y sociocultural de los estudiantes, desde sus respectivos lugares de apropiación del sistema educativo. Ahora bien, esto implica que los textos han de considerarse más que bastas herramientas, al respecto, señala D’Alton

Los materiales didácticos en general se consideran como meros instrumentos: se evalúan por su supuesta capacidad para producir determinada reacción o modificación

4 Con el desarrollo en el uso de las Tecnologías de la Información y la Comunicación (TIC) en la educación a distancia, la literatura reciente en esta área se ha inclinado hacia estudios centrados en el aprendizaje en línea o basados en la web. Sin embargo, la investigación sobre materiales escritos, particularmente sobre formas de alcanzar metas en la formación de profesionales de la Educación, no deberían ser descuidados en una época de aumento en el uso de educación a distancia para la formación docente. (traducción libre) 
en el alumno y se les acomoda dentro de un esquema de valores derivado de este contexto externo, nunca surgido de su propia naturaleza lingüística. (1994, p. 1)

Al ser la comunicación un proceso complejo, cabe cuestionarse cuál es la posición que se ha de tomar respecto a la mediación pedagógica, como base del proceso mencionado. La pedagogía, desde su significado originario, da relevancia a la característica de acompañamiento ${ }^{5}$; en el caso particular de la educación a distancia, los materiales didácticos cumplen la función de acompañar durante el aprendizaje.

Naranjo (2010), al respecto, considera que la comunicación acepta una "interacción permanente, sincrónica o asincrónicamente, entre el alumno, los especialistas y los materiales didácticos y facilitar la retroalimentación"; lo cual refuerza la necesidad de identificar -en las diferentes etapas de la producción- situaciones que permitan promover y reforzar el aprendizaje, recurriendo a la adecuación del lenguaje al contexto de uso, a la comprensión de lo que se ha concebido como el modelo pedagógico y la función del productor académico como especialista en el léxico propio de su área de conocimiento.

Finalmente, el objetivo principal de este texto será demostrar, en la educación a distancia, la preponderancia de la adecuación del lenguaje de los materiales didácticos escritos $^{6}$, al contexto de los estudiantes, en el proceso de producción como proceso pedagógico.

\section{Adecuación, contexto y mediación}

El lenguaje se caracteriza de maneras diferentes según la perspectiva teórica desde la cual se analice; esta diversidad ha de tomarse en cuenta cuando se identifica que, precisamente, el objeto con el cual se trabaja en la producción y mediación del contenido de las unidades didácticas es escurridizo, flexible y con la capacidad de permitir soluciones creativas en vez de recetas o esquemas inamovibles.

En similares circunstancias nos encontramos ante la dificultad que plantea tratar de definir el concepto de situación comunicativa en la educación a distancia, pues, como explica

5 A sabiendas de la variedad de materiales que pueden encontrarse bajo la etiqueta de "materiales didácticos", se aclara que en este ensayo solamente se hace referencia al material escrito impreso (unidades didácticas, guías de estudio, material complementario) y en algunos casos digital, que se produce en Promade.

La palabra proviene del griego maıðaүwyós (paidagogós), en sentido estricto, era la persona -el esclavoencargada de llevar a los niños de la mano. De las raíces "paidos" que significa niño y "ago", llevar o conducir. Por extensión, se llama así a los maestros que guían al niño en lo relativo a su educación. 
García (1998), inclusive dentro del aula es metodológicamente insuficiente, en especial cuando se plantea "como el conjunto de elementos contextuales que determinan la conversión de una textura en un texto dentro de un acto semiótico verbal" (p.129).

Por lo que, únicamente ubicándonos en la perspectiva de la producción de textos, nos daríamos cuenta de que la realidad que se trata de suplantar en el lenguaje escrito debe proveer la mayor cantidad de texturas completas para generar texto, debido a su incapacidad para reproducir presuposiciones cognoscitivas y pragmáticas de emisores y receptores reales (García, 1998), es decir, la aplicación del lenguaje como ejercicio pedagógico de la escritura permitirá la generación de conocimiento, dentro de una situación específica que lo envuelve.

En suma, en este espacio se han considerado algunas caracterizaciones de diversos planteamientos teóricos para el tratamiento de estos tres conceptos, de tal manera que puedan tomarse como puntos de partida para conjuntar la tríada adecuación-contextomediación.

\subsection{Situación comunicativa y adecuación del lenguaje}

Inicialmente, ha de indicarse que el lenguaje está considerado como uno de los aspectos que integran la mente humana. Es visto como un instrumento de capacidad cognoscitiva y afectiva del individuo; un conjunto de signos lingüísticos que posibilitan la expresión y la comunicación humana (Piera, Chomsky y Díez, 2002). Para Lyons (1984), este es un sistema de códigos con cuya colaboración se designan los objetos del mundo exterior, sus acciones, cualidades y relaciones entre ellos.

El lenguaje no es homogéneo ni uniforme, sino que muestra diferenciaciones según diversos factores: el tipo de educación, el canal de comunicación, la interrelación entre los hablantes, la situación de comunicación, el grupo social, la historia, la geografía, ente otros (Escandell, 2005).

Por lo tanto, adecuar el lenguaje significa saber elegir, de entre todas las soluciones lingüísticas que da la lengua, la más apropiada para cada situación de comunicación. Es decir, es necesario escoger un tipo de estrategia educativa, a distancia; adecuar el currículum de un determinado nivel educativo, con el propósito de hacer que ciertos objetivos o contenidos sean más accesibles a un alumno o un determinado grupo de personas ubicado en diferentes espacios geográficos. 
Para Bruner (2001, p. xx), "se trata de tener en cuenta las limitaciones del alumno a la hora de planificar la metodología, los contenidos y, sobre todo, la evaluación". Se pretende que la adecuación del lenguaje sea, a la vez, analítica y sintética, concreta y abstracta, puntual y general.

Desde esta perspectiva, el lenguaje es un sistema de signos que codifica los pensamientos que un emisor concibe en su interior y los transmite a un destinatario, quien los descodifica y así los hace inteligibles a su propia psique; así, la adecuación del lenguaje se concibe en función de la situación comunicativa. Sin embargo, también existe otra línea de pensamiento lingüístico que ve el lenguaje como molde que conforma la cognición y hasta cierto punto- la percepción, y como herramienta cognitiva forjada en la dinámica de la interacción social.

En esta línea de pensamiento podemos colocar a Vygotsky (constructivismo dialéctico), quien considera que el medio social es crucial para el aprendizaje, producido por la integración de los factores sociales y el personal. Asimismo, Sapir y Whorf, en su hipótesis de trabajo, establecen que existe una cierta relación entre las categorías gramaticales del lenguaje que una persona habla y la forma como la entiende y conceptualiza el mundo. De igual manera, se manifiesta en el trabajo sobre la categorización de Lakoff, quien a través de su teoría sobre el pensamiento metafórico nos permite estructurar conceptos a partir de otros y la forma de realizar este proceso depende de nuestra experiencia directa del mundo. Otros pensadores modernos, entre ellos John Ellis, mantienen que el corazón del lenguaje es la categorización. Desde esta perspectiva, el lenguaje cobra aún más relevancia en los procesos didácticos, no solo por ser connatural del pensamiento sino por su papel en los conceptos y en la abstracción ${ }^{7}$.

Desde luego, la adecuación del lenguaje no es posible establecerla como una receta que sea aplicable a cualquier texto, de cualquier carrera profesional, por ello, debe tenerse clara la situación comunicativa -a la cual se deberá adaptar el contenido posteriormentepara que se den los resultados esperables, entre ellos, en primera instancia, el establecimiento de una relación dialógica entre el estudiante y la unidad didáctica (cuya meta es tener mayor relevancia que la figura del profesor del sistema presencial). Así, se

7 En estos dos párrafos se mencionan las diversas líneas teóricas relacionadas con el lenguaje; sin embargo, no serán tratadas en este documento. 
conceptualiza como el conjunto de elementos, tanto lingüísticos como extralingüísticos, presentes al momento de la comunicación.

Imaginar, entonces, la particular situación comunicativa de los estudiantes a distancia implica que, para adecuar los textos, el autor ha de identificar un acto semiótico en el cual el alumno pueda desarrollar la capacidad de leer un documento que le vaya dirigiendo, paso a paso, de manera individual y personalizada, en el proceso de adquirir y reflexionar sobre el conocimiento; asimismo, el lenguaje deberá ser formal, pero sin perder la claridad y la posibilidad de ser comprendido por un público amplio, cuyo rango de edades es variable y su ubicación geográfica diversa.

De esta manera, uno de los desafíos más importantes de la educación a distancia es la elaboración de material didáctico que sea realmente de aprendizaje interactivo, lo cual implica:

- Brindar contenidos significativos, motivantes, pertinentes y actualizados dentro de una estructura dinámica.

- Provocar, prever y proveer estrategias cognitivas que sean para el sujeto factor de desarrollo posterior por sí mismo.

- Fortalecer el diálogo, a través de diversas actividades didácticas inteligentes capaces de desafiarlo, con el tutor, con otros estudiantes, usando tecnología apropiada que lo facilite.

- Seleccionar y organizar códigos adecuados en coherencia con los objetivos didácticos y el contenido, a fin de brindar las condiciones óptimas para su codificación y descodificación (Manzoor y Raham, 2011; Murillo, s.f.).

Así, la interactividad en la educación a distancia se alimenta de los contenidos de textos procesados didácticamente, o sea, de la elaboración de materiales bien estructurados.

Por último, el reconocimiento del contexto y las características de la población coadyuvan en la disposición de los diversos materiales; como bien lo describe Méndez (2007, p. 4), a la condición en "solitario", en la que el estudiante se enfrenta al texto, también se une el cansancio, porque estos alumnos suelen trabajar, y su poco tiempo libre lo dedican al estudio.

En el estudio titulado Factores que inciden en el rendimiento académico y la permanencia de los estudiantes en la UNED (Barahona y Rodino, 2009), investigación elaborada con una muestra de 1182 estudiantes activos de la UNED en el 2004, se fortalece 
la hipótesis de que la capacidad para escribir un texto bien estructurado está correlacionada positivamente con el rendimiento académico.

\subsection{Cómo se concibe el lenguaje escrito en las producciones académicas}

Al explorar la situación comunicativa y la adecuación del lenguaje, es necesario imaginarnos lo que un estudiante a distancia hace cuando aprende y lo que el texto, unidad didáctica en nuestro caso particular, efectúa cuando enseña. Además, es preciso subrayar que a la unidad didáctica le corresponde asumir todo el peso de la enseñanza aprendizaje vigente, pues el texto es una unidad comunicativa, es un mensaje objetivo en forma de documento escrito que consta de una serie de enunciados unidos mediante diferentes enlaces de tipo gramatical y lógico (Rodríguez, 1997).

Por consiguiente, la creación de un texto escrito se concibe como un proceso complejo en el que intervienen, de manera interrelacionada, factores culturales, sociales, emotivos o afectivos, cognitivos, físicos (viso-motores), discursivos, semánticos, pragmáticos y verbales (Rodríguez, 2001).

El enfoque didáctico en la producción de textos en la educación a distancia resalta la importancia de los componentes cognitivos (planificación, producción y revisión del texto) intervinientes en la composición escrita y su relación con los factores sociales y culturales, los cuales se desenvuelven en un espacio y un tiempo específico, sin dejar de mencionar los componentes metacognitivos que influyen en el desarrollo de la composición escrita: el carácter afectivo y emocional (Bruning y Horn, 2000).

Recordemos que el lenguaje, creado como una totalidad en sí mismo, no se puede separar del contexto en el cual se utiliza; sin embargo, para efectos de análisis de la información, sistematización, recolección y claridad conceptual está compuesto por cuatro prácticas: oralidad, escritura, lectura y literatura (Piera et al., 2002). No se debe perder de vista que estos componentes se influyen entre sí y están en una constante interrelación; sin embargo, en este ensayo tomaremos en cuenta la escritura, ya que es la práctica presente en las unidades didácticas de la educación a distancia.

Cuando se lee un texto, el alumno interactúa con este y pone en juego sus diferentes habilidades: el análisis, la reflexión, el contraste con saberes previos y la selección de información relevante. Por lo tanto, un mismo escrito puede tener diversas interpretaciones facilitadas por los lectores y no una sola forma de comprenderlo. 
De igual manera, es significativo tomar en consideración cómo la lectura, al igual que la escritura, es una práctica del lenguaje permitida en situaciones concretas con el fin de facilitar la comunicación entre los individuos de una sociedad. Sucede entonces en el marco de la vida social y es una herramienta de vinculación con esta; de ahí, se colige la trascendencia del conocimiento de la situación real de estudio, es decir, al tener certeza de lo que se necesita, el discurso al cual se recurre permite al escritor ser pertinente en su intervención, pues, en circunstancias ideales, este debería "conocer la materia, escribir correctamente, saber enseñar y estar adaptado al sistema a distancia" (Murillo, s. f., p.7).

Aunado a ello, la mediación pedagógica es una característica esencial, tanto de la educación a distancia como del material de apoyo ofrecido al estudiantado, ya que al diseñar los medios para los distintos tipos de aprendizaje habrá "que imaginar metodologías para acercar los esquemas de conocimientos previos a conjuntos diversos y flexibles de conocimientos, cada vez más cambiantes" (UNED, 2007, pp. 29-30).

El primer acercamiento a esta mediación corresponde a uno de los procesos básicos del aprendizaje: la comunicación; de tal manera, "la comunicación didáctica se concibe como el proceso o recurso necesario con el que se logran las estrategias de mediación para el desarrollo del lenguaje y de la cognición" (UNED, 2007, p. 41). Esto evidencia el carácter interactivo del material didáctico y "la participación activa del alumno como protagonista del proceso de enseñanza y aprendizaje" (UNED, 2007, p. 41).

Es así como, en este engranaje representado por el ajuste del lenguaje, el contexto y la mediación, entra la producción de material didáctico, que conlleva un importante trabajo en equipo, concentrado en la imagen del productor o editor académico, con la participación activa de los interesados en la calidad del material por producir: encargado de cátedra, de programa, autor, en algunos casos, los tutores y alumnos, principalmente cuando evalúan el material.

\subsection{Proceso de producción, proceso pedagógico}

Al pensar en el proceso de producción, normalmente se tiene la idea de que este podría ser engorroso y hasta inútil, de no ser porque una de las misiones de la Universidad es producir el material propio, original, que se utiliza en los diversos cursos presentados en la oferta académica. Para tenerlo un tanto más claro, se presenta a continuación un esquema correspondiente a las etapas básicas de producción: 


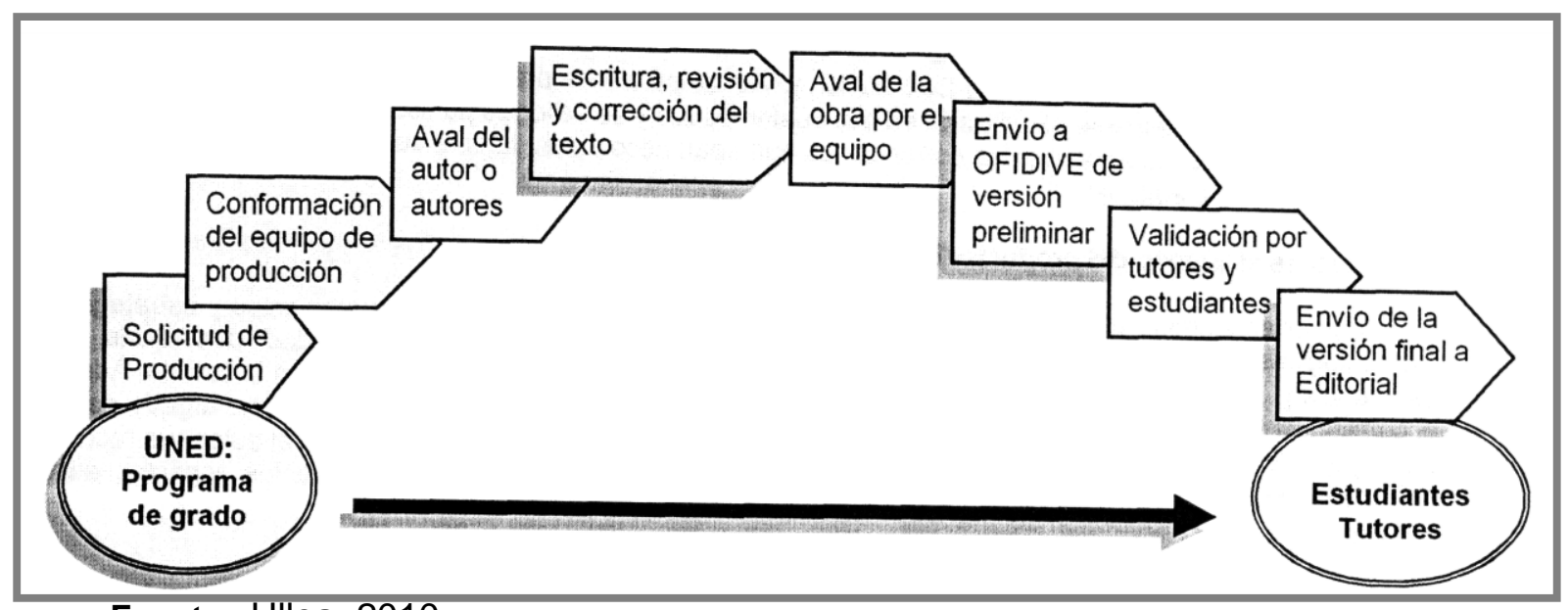

Fuente: Ulloa, 2010

Específicamente, para producir las unidades didácticas, antologías y guías de estudio se ha de comprender que los trámites, desde la solicitud hasta la impresión final, permiten crear o renovar el material y acercar los contenidos a la realidad del estudiante, pues lo esperable es que los autores se dispongan a mostrar hechos de la sociedad costarricense en el discurso escrito.

Los participantes directos de este proceso de producción son: el autor, los encargados de cátedra y programa, el especialista y el editor académico. Cada uno se compromete a asumir el papel que debe desempeñar (en este caso, se está tratando únicamente la función del editor); pero, también, se requiere identificar e interiorizar el concepto de mediación pedagógica, para cuando sea necesario aplicarlo con el fin de conjuntar elementos como los objetivos de aprendizaje, la descripción general de contenidos, las estrategias metodológicas, la evaluación y su relación con los medios y recursos didácticos.

La función del productor académico es la de filtrar la información que se proporcionará al estudiante; sin embargo, al estar especializado en una determinada área académica, cada editor adquiere una perspectiva diferente del lenguaje que debería utilizarse, por ello, unificar el sistema de escritura de las unidades didácticas se complica ante variables como la carrera, la población, la cantidad de alumnos y los objetivos planteados en la descripción curricular para determinada materia.

Ante esto, lo recomendable para cumplir con la adecuación pragmática de un texto es comprender el discurso en su significación más amplia, apunta Gee (en Reed, 2005, p. 271), 
ya que este es una especie de kit de identidad, lo cual permite seguir una serie de instrucciones acerca de cómo actuar, hablar y escribir, con el fin de que los demás puedan reconocer un determinado papel.

A lo anterior, también, se le denomina "identidades prácticas" que, referidas a un contexto determinado de una actividad específica, asignan un valor a ciertos actos; precisamente, el dar una posición relevante a tópicos, objetivos y actividades es una tarea que lleva a cabo el productor académico desde la ideología particular de su propia carrera profesional.

La calidad del texto dependerá entonces de la perspicacia y el dominio de la materia del editor académico para encontrar el léxico, la sintaxis y el estilo que presenten el conocimiento, plasmado por el autor, nítidamente; así, el aprendiente obtiene un insumo lingüístico, que le permite adaptarse y adaptar el saber, desde un cuestionamiento y un proceso de reflexión. A su vez, se reafirma lo planteado en el Modelo pedagógico de la Universidad Estatal a Distancia, acerca de que "toda construcción cognitiva tiene lugar primero en el cuerpo social y es interiorizada luego por el individuo e incorporada a sus estructuras de conocimiento" (2007, p. 34).

Finalmente, la relevancia de considerar la adecuación del lenguaje, como parte de los aspectos fundamentales de la mediación pedagógica, no es la de presentar el contenido superficialmente, sino la de facilitar el acceso y promover la motivación para aprehender el conocimiento a partir del diálogo con el texto y sus múltiples voces.

\section{A manera de conclusión (...y recomendaciones)}

Es claro que el medio inicial para transmitir conocimientos, a nivel simbólico, es el lenguaje, por lo que su adecuación a un contexto es fundamental para dar los primeros pasos en el proceso de aprendizaje.

La consideración del concepto de mediación pedagógica implica, además, la comunicación como proceso complejo, con una posición específica como guía, por ende, relevante y con la función de facilitar la interacción y la retroalimentación.

La adecuación del lenguaje se encuentra estrechamente relacionada con la situación comunicativa, en este caso, aquella en la cual se encuentra inmersa la interrelación la establecida entre el material didáctico y el estudiante, lo cual determina que, para obtener resultados favorables en este tipo de educación, se debe establecer una relación dialógica. 
Dicha relación permite, a su vez, conocer la situación real de estudio y el diseño y aplicación de metodologías para ofrecer, según los tipos de aprendizaje; el acercamiento a esta realidad social, por parte de encargados y productores académicos, permite flexibilizar el material, de tal manera que se contribuya a la actualización de los contenidos, según los requerimientos de la población estudiantil.

El proceso de producción de materiales didácticos tiene la función de contribuir en la adquisición de conocimientos, tanto para los estudiantes como para los autores y demás participantes. Esto sería eficaz si se unificara el concepto de mediación pedagógica, los procesos se agilizaran y surgiera la posibilidad de enseñar a los interesados a plasmar sus conocimientos, de manera que un lenguaje técnico, por ejemplo, pueda escribirse transmitiendo la información requerida, permitiendo al alumno identificarse con la materia y enseñándole a reflexionar y tomar una postura crítica, la cual le permita dar respuestas al medio.

A lo largo de este ensayo, se hace evidente la preocupación por reconocer, también, al usuario del texto, ya que, sin importar cuánto contenido teórico se maneje, es necesario que el editor académico sea capaz de observar y conocer el contexto donde se desenvuelve el alumno, de manera tal que su intervención en el proceso de producción sea fundamental para proyectar, por medio de la filtración del discurso y la mediación a su cargo, los conocimientos de un área específica de forma práctica, clara y adecuada.

Consideramos, además, que la imposibilidad de establecer un "recetario" para la producción de material didáctico escrito -únicamente tomando en consideración el nivel del lenguaje escrito- permite enriquecer los textos, hacer más agradable el aprendizaje y acercar al estudiante a la realidad académica en la cual se circunscribe, contribuyendo a desarrollar su capacidad para leer e interpretar, reconociendo las múltiples voces que permean el discurso pedagógico y, al mismo tiempo, apropiándose de estas mediante el análisis.

\section{Referencias}

Barahona, Mario y Rodino, Ana María. (2009). Factores que inciden en el rendimiento académico y la permanencia de los estudiantes en la UNED. San José, Costa Rica: Universidad Estatal a Distancia.

Bruner, Jerome. (2001). El proceso mental en el aprendizaje. Narcea: Madrid. 
Bruning, Roger y Horn, Christy. (2000). Developing motivation to write. Educational Psychologist, 35 (1), 25-37.

Castiñeiras, Martha. (2003). Pedagogía, conocimiento crítico y multiculturalismo. Un desafío para el siglo XXI. Acción pedagógica, 12 (1), 16-19.

D’Alton, Cristina. (1994). El análisis del discurso y los materiales didácticos. San José, Costa Rica: Editorial Universidad Estatal a Distancia.

Escandell, María. (2005). La comunicación. Gredos: Madrid.

Fung, Yvonne. (2005). Teacher's views on and use of printed materials in a distance learning teacher education course. Open Learning, 20 (2), 175- 183. Recuperado el 4 de setiembre de 2010 de la base de datos EBSCOHost.

García, Fernando. (1998). El aula y la situación comunicativa (ASELE Actas I, pp. 129135). Recuperado el 5 de setiembre de 2010, de http://cvc.cervantes.es/ensenanza/biblioteca ele/asele/pdf/01/01 0277.pdf.

Lyons, John. (1984). El lenguaje y la lingüística. Teide: Madrid.

Manzoor, Muhammad y Rahman, Fazalur. (2010). Impact of theories of distance education on teaching learning process. International Journal of Academic Research, 2 (4), 373- 378. Recuperado el 4 de setiembre de 2010 de la base de datos EBSCOHost.

Méndez, Sylvia. (2007). La estrategia de entrada en la mediación pedagógica. Posgrado y sociedad, 7 (2), pp. 1 - 20.

Murillo, Olga. (s.f.). Autores de la UNED: Adecuación a metodología a distancia. Innovaciones educativas. 6 (10), 61-65. Recuperado de http://www.uned.ac.cr/globalnet/global/ensenanza/entrega/artículos/auto resdelauned.htm

Naranjo, Gustavo. (2010). Los materiales didácticos en la UNED. Pedagogía universitaria en la educación a distancia [Diapositivas. Presentación Power Point]. Universidad Estatal a Distancia: San José, Costa Rica.

Piera, Carlos, Chomsky, Noam y Diez, José. (2002). El lenguaje y la mente humana. Barcelona: Ariel.

Reed, Yvonne. (2005). Using students as informants in redesigning distance learning materials: possibilities and constraints. Open Learning, 20 (3), 265- 275. Recuperado el 4 de setiembre de 2010 de la base de datos EBSCOHost.

Rodríguez, Mauro. (1997). El pensamiento creativo. México: Editorial MC Graw Hill.

Rodríguez, Mauro. (2001). Algunas consideraciones acerca de la producción de un texto escrito. Islas, 43 (129), 52-63. 
Ruiz, Pilar y Escandell, María Victoria. (2009). El lenguaje humano.: Madrid: Editorial Universitaria Ramón Areces.

Thinès, Georges y Lempereur, Agnes. (1978). Diccionario de ciencias humanas. Madrid: Ediciones Cátedra.

Thurn, David. (1999). A Comparative Report on Writing Programs. Princeton, N.J.: MimeoPrinceton Writing Program.

Ulloa, Gilbert. (2010). Producción de materiales didácticos impresos. Proceso de inducción (Promade). San José, Costa Rica: Universidad Estatal a Distancia.

Universidad Estatal a Distancia - UNED. (2007). Modelo pedagógico de la Universidad Estatal a Distancia (2. a reimp.). San José, Costa Rica: Editorial Universidad Estatal a Distancia (EUNED. 